\title{
Le deuil du conjoint en pays évhé
}

Ewe Mourning Rites (Togo)

\section{Albert de Surgy}

\section{OpenEdition}

\section{Journals}

Édition électronique

URL : http://journals.openedition.org/span/1138

DOI : $10.4000 /$ span. 1138

ISSN : 2268-1558

\section{Éditeur}

École pratique des hautes études. Sciences humaines

\section{Édition imprimée}

Date de publication : 1 novembre 1989

Pagination : 105-134

ISSN : 0294-7080

\section{Référence électronique}

Albert de Surgy, «Le deuil du conjoint en pays évhé », Systèmes de pensée en Afrique noire [En ligne], 9 | 1989, mis en ligne le 15 octobre 2013, consulté le 10 décembre 2020. URL : http:// journals.openedition.org/span/1138; DOI : https://doi.org/10.4000/span.1138 


\title{
LE DEUIL DU CONJOINT EN PAYS EVHE
}

\author{
par
}

\section{Albert de Surgy}

On emploie le mot deuil pour désigner aussi bien le douloureux désarroi de celui qui vient de perdre un de ses proches que, de façon plus objective, l'ensemble des mesures par lesquelles la société en prend acte et en dirige l'expression, ne le rendant ainsi évident que pour mieux y mettre fin. Ne nous hasardant pas à analyser les sentiments éprouvés par des Africains à la suite d'une mort, nous nous bornerons à étudier les rites auxquels les Evhé (répartis à proximité du littoral des deux côtés de la frontière séparant le Ghana du Togo) soumettent traditionnellement ceux des leurs qu'ils estiment en être les plus affectés. Ces rites nous permettront toutefois de mieux comprendre la façon dont est perçue, et en conséquence ressentie chez eux, la disparition de tel ou tel.

Nous excluerons du champ de nos réflexions toutes les cérémonies funéraires (traitement du cadavre, enterrement, premières funérailles, secondes funérailles, offrandes au mort et à ses compagnons invisibles) qui ne se proposent pas ouvertement de remédier aux conséquences de la rupture soudaine des liens qui unissaient un défunt à un ou plusieurs de ses proches. Exécutées pour faciliter l'insertion dans l'au-delà de l'âme du mort, ces cérémonies sont certes de nature à rassurer les survivants comme à leur donner bonne conscience et contribuent donc à les apaiser. Il n'empêche que l'appellation de rites de deuil mérite à notre avis d'être limitée au Systèmes de pensée en Afrique Noire, 9, 1989 
petit nombre de ceux qui, au lieu d'être dédiés à un mort, apparaissent centrés sur des personnes qui se voient mises dans l'obligation de respecter temporairement certaines règles (vestimentaires, alimentaires et de comportement) destinées à les préserver d'effets malsains. Alors seulement pouvons-nous parler de prise de deuil.

Trois sortes de rites de deuil, entendus en ce sens restreint, peuvent être observés chez les Evhé. Ils correspondent à une distinction, dont j'ai déjà signalé l'existence chez les Mwaba-Gurma (Surgy, 1983, p. 37-38), de trois types de relations unissant par paires six termes primordiaux rapportés d'une part aux six extrémités du corps humain (pieds, mains, tête et sexe), d'autre part à six strates célestes dont le parcours dans les deux sens par la lune définit une "année lunaire" de douze lunaisons.

Les premiers concernent une rupture des relations établies entre deux rivaux de même rang, deux partenaires d'un même jeu, deux sources d'une incessante dialectique. Ils sont exécutés après la mort du premier membre d'un couple de jeunes jumeaux. Jusqu'à leur septième année environ, ceux-ci sont jugés à ce point inséparables que le survivant risquerait de suivre le premier dans la mort si celle-ci ne se trouvait pas symboliquement niée. A cet effet une statuette de bois représentant le défunt est confectionnée et l'on en prend soin comme s'il s'agissait d'un véritable enfant. Plutôt que d'écraser celui que le mort a épargné par un "travail de deuil" qu'il ne serait sans doute pas assez robuste pour supporter, on préfère l'inviter à épuiser ainsi en imagination tous les rapports qu'il aurait souhaité ne jamais cesser d'entretenir avec celui ou celle qui lui fait défaut (cf. Surgy, 1988 : 278-279).

Les seconds concernent au contraire une rupture des relations établies entre deux personnes dont l'une domine hiérarchiquement l'autre, tout en l'aidant bienveillamment à s'accomplir. Ils sont exécutés pour quelqu'un après la mort d'un parent, d'un aîné ou d'un maître spirituel. Débutant, à la discrétion des intéressés, à une date parfois très largement postérieure à l'issue des funérailles qui sont elles-mêmes assez distantes des cérémonies qui entourent l'enterrement, ils consistent en l'adoption solennelle temporaire, modérément 
sujet dans Rivière, 1982). Je me contenterai de décrire les cérémonies qui étaient encore célébrées récemment à Anloga, capitale du pays anlo, car elles sont apparemment les plus riches; je signalerai en notes quelques variantes attestées dans le voisinage; je ferai état ensuite des principales différences entre les cérémonies observées à Ave-Dzolo et celles d'Anloga.

\section{1 - LES CEREMONIES DU VEUVAGE CHEZ LES ANLO}

A Anloga ce sont deux femmes appartenant à la famille paternelle et à la famille maternelle du défunt (et non du survivant) qui sont invitées à effectuer les cérémonies, aussi bien pour un veuf que pour une veuve ${ }^{1}$. Il suffit qu'elles soient au courant du rituel, car il n'y a pas pour cela de spécialistes attitrés.

Examinons tout d'abord le traitement réservé aux veufs avant d'expliquer comment s'en distingue celui des veuves.

\section{Cas du veuf}

a) Réclusion à domicile

Jadis le veuf était confiné chez lui pour une durée théorique de huit jours (limitée en fait à sept nuits et six jours). Il commençait par être confiné dans sa chambre pour une durée théorique de quatre jours (limitée en fait à trois nuits et trois jours). Puis, à compter du quatrième jour, il demeurait confiné pendant une durée théorique de quatre autres jours (en fait quatre nuits et trois jours) dans l'ensemble de sa maison, pouvant dès lors occuper aussi sa pièce de séjour. En considérant comme premier jour celui où on l'avait introduit dans la chambre, on venait mettre fin à sa réclusion le matin $\mathrm{du}$ huitième jour.

Depuis quelques décades le veuf n'est plus confiné chez lui que pour une durée théorique de quatre jours (limitée en fait à trois nuits et deux jours). Dès le lendemain de son introduction dans sa

1 Selon G.K. Nukunya (1969, p. 206) qui se réfère aux coutumes en vigueur à Woe (pays anlo), ce sont un ancien veuf et une ancienne veuve qui officient, le rôle principal étant tenu par celui de même sexe que le sujet. 
chambre on lui permet de passer de celle-ci dans sa pièce de séjour, et on vient mettre fin à sa réclusion au matin du quatrième jour.

L'introduction dans la chambre a toujours lieu le soir d'un ketasigbe, jour du marché à Keta et Anloga, jugé propice au culte des ancêtres.

Le soir venu, les deux officiantes versent d'abord des libations d'eau enfarinée et d'alcool à l'intention de l'épouse défunte, lui déclarant que son mari va faire les cérémonies d'aho. Après avoir rasé elles-mêmes le veuf à l'intérieur de sa cour, elles le conduisent devant la porte de ses appartements, le saisissent par la tête et le "comptent" jusqu'à quatre ${ }^{2}$ avant de le faire entrer dans la pièce de séjour. Puis elles répètent les mêmes gestes avant de l'introduire dans sa chambre à coucher.

Là elles lui remettent un à un, en comptant encore chaque fois jusqu'à quatre, tout ce qu'il lui a fallu se procurer pour assumer sa condition de veuf reclus:

- des haillons sous lesquels il ne devra porter qu'un cache-sexe (bande d'étoffe retenue par-devant et par-derrière par une corde passée autour des reins),

- une natte où s'étendre pour dormir,

- une assiette en argile pour y recevoir de la viande ou du poisson en sauce,

- une calebasse couverte (akpakuvi) pour y recevoir de la pâte de maïs (akplê),

- une petite calebasse neuve pour boire de l'eau,

- une éponge et du savon pour se laver.

Puis elles font semblant de lui raser encore trois fois la tête (cela en mémoire du temps où le veuf demeurait confiné quatre jours dans sa chambre et où elles revenaient chaque jour lui raser la tête de frais jusqu'au soir du quatrième jour avant de l'introduire dans la pièce de séjour).

\footnotetext{
2 Les comptages rituels sont très fréquents chez les Evhé. L'officiant se saisit à deux mains d'un objet ou d'une personne et lui imprime, dans telle ou telle direction (point cardinal, lieu précis, destinataire) un nombre déterminé d'impulsions (le plus souvent 3, 4 ou 7 ) en nommant successivement les chiffres correspondants $(1,2,3 \ldots)$.
} 
Dès lors, le veuf ne doit plus sortir de chez lui si ce n'est pour ses besoins naturels ${ }^{3}$ ou pour aller discrètement se laver, avant tout le monde au point du jour et après tout le monde la nuit venue. Un ancien veuf ou une ancienne veuve de sa propre famille paternelle vient lui apporter à manger et à boire et reste non loin de là à sa disposition au cas où il aurait besoin de quelque chose.

Pour passer le temps il tresse des fibres ededho, provenant des ramures du palmier à huile, avec lesquelles il confectionne de la ficelle et des cordes qui seront ensuite vendues pour son profit personnel ( $s^{\prime}$ il ne sait pas encore tresser des cordes, un veuf ou une veuve viendra le lui apprendre).

Seuls d'anciens veufs ou d'anciennes veuves ont le droit de lui rendre visite et de discuter un peu avec lui. Ils frappent deux fois à la porte et ne doivent pas l'appeler par son nom, mais crier: "Aho!" . Le veuf ne doit pas leur dire d'entrer, mais frapper un nombre indéterminé de fois sur un objet quelconque de sa chambre (son lit, ou sa table, par exemple) pour leur montrer qu'il est bien là et les inviter à ouvrir. Il ne doit manifester sa présence à la suite d'aucun autre appel, même de ceux qui émanent de membres très proches de sa famille.

b) Premier lavage et remise du pagne de deuil noir

Le quatrième jour avant l'aube (autrefois le huitième jour), nécessairement un ashimlegbe, ou veille du marché de Keta) ${ }^{4}$, vers 4 heures $30 \mathrm{du}$ matin, les deux femmes qui ont introduit le veuf dans sa case viennent l'en faire sortir.

Devant la porte de la case où se tient le veuf, elles prient tout d'abord, l'une après l'autre - celle qui appartient à la famille paternelle de l'épouse défunte en premier, celle qui appartient à la famille maternelle en second. S'adressant à la défunte, elles déclarent

\footnotetext{
3 Pour lui éviter d'avoir à sortir de chez lui dans la journée pour ce motif, on lui aménage parfois un trou dans son enclos de toilette. Ailleurs, comme à Atorkor, on tolère qu'il sorte à condition qu'il avance en tenant ses deux mains posées sur ses épaules, les coudes appuyés contre sa poitrine.

${ }^{4}$ Ce jour est propice aux relations avec les esprits et les divinités. A Atorkor on préfère fixer réellement le nombre de nuits de retraite du veuf ou de la veuve à un multiple de quatre et on introduit en conséquence le veuf ou la veuve en retraite un ashimleqbe.
} 
"- X ..., aujourd'hui nous allons sortir ton époux. Apporte du bien à ton époux ...", et elles versent une libation d'eau enfarinée.

Ceci fait, elles frappent deux fois à la porte de la chambre et appellent : "-Aho !". Le veuf leur répond en prenant un objet quelconque pour frapper le sol ou un meuble. Alors les officiantes ouvrent la porte. Elles le prennent ensemble, à deux mains, le tournent vers l'est et vers l'ouest en prononçant les paroles d'usage ${ }^{5}$, puis le "comptent" quatre fois et le font sortir dans la pièce de séjour, puis dehors .

Elles l'emmènent au bord de la mer ${ }^{6}$ en compagnie d'une ou deux autres personnes de sa famille. C'est toujours au lieu-dit Godzomonu que les habitants d'Anloga emmènent les veufs pour le lavage rituel. Sur le chemin qui y conduit il y a huit emplacements précis, le dernier à la plage, où il convient de prier. A cet effet on prend soin d'emporter un seau d'eau enfarinée, à verser sous forme de libations, et huit cauris. A chaque emplacement les officiantes élèvent une butte de terre (ou de sable) et déposent à son sommet un cauri. Elles prient à tour de rôle, la veuve appartenant à la famille paternelle de la défunte en premier, celle appartenant à la famille maternelle en second. Ayant présenté à l'est puis à l'ouest, en prononçant les paroles d'usage, une calebasse d'eau enfarinée, elles demandent la protection de Dieu et des ancêtres, déclarant, par exemple, à l'épouse défunte : "Nous conduisons ton mari à la mer. Il faut que la mer soit calme (litt. "sans creux") pour le laver". Puis elles comptent trois fois la calebasse et versent la libation en trois fois au pied de la butte.

Ayant répété ceci huit fois, et étant arrivées à la plage, elles rasent à nouveau le veuf (cheveux, poils axillaires et poils pubiens) et lui retaillent les ongles. Puis elles creusent dans le sable, au plus près des vagues, une cavité qui se remplit d'eau de mer. Elles aspergent le veuf d'eau de mer, le prennent à deux mains, par les épaules, le "comptent" quatre fois et lui font prendre place, debout,

\footnotetext{
5 Vers l'est : "Voici pour les mauvais (bis). Si le soleil se lève, qu'il se lève sur eux!" Vers l'ouest : "C'est pour nous le soir (bis). Un enfant du soir va jusqu'au soir".

'Dans les villages éloignés du rivage maritime les veufs sont généralement emmenés, pour être lavés, à un croisement de deux chemins.
} 
dans la cavité. Le veuf s'y lave lui-même en utilisant du savon et une éponge. S'il le désire il peut avancer dans les vagues et même nager quelques instants ${ }^{7}$.

Toujours vêtu de ses haillons, le veuf revient ensuite vers les officiantes qui lui confient habituellement pour se rincer un pot d'eau douce. Ceci fait, elles lui ôtent ses haillons et son cache-sexe. Puis, agissant toujours ensemble, ayant présenté chacun de ces objets à l'est et à l'ouest en prononçant les paroles d'usage, et les ayant comptés quatre fois, elles lui remettent un cache-sexe neuf et un pagne de deuil noirs ${ }^{8}$ dont il s'habille pour revenir à la maison.

Avant le départ elles enterrent dans le sable les haillons et le cache-sexe que portait le veuf, les poils, les cheveux et les ongles qu'elles lui avaient coupés en arrivant à la plage, enfin l'éponge et la vieille serviette dont il s'était servi pour se laver.

Il pourra désormais, mais en tenue de deuil, vaquer librement à ses occupations, et dormir et manger comme il lui plaira. Cependant il lui sera encore interdit de se mêler activement à des réjouissances publiques et de chanter ${ }^{9}$.

La natte sur laquelle il dormait durant les nuits de sa réclusion devient la propriété de l'officiante appartenant à la famille paternelle de sa défunte femme. Quant à l'écuelle et aux calebasses dont il se

7 Le lavage d'un veuf ne fait intervenir aucune plante magico-médicinale (ama). Cependant, l'eau de mer est une eau à laquelle on attribue un caractère "chaud" ( douce avec laquelle il se rince habituellement après coup est une eau de caractère "frais" (fafa). Ce n'est que dans les villages de l'intérieur qu'on éprouve parfois le besoin de rendre "chaude" et "fraîche" l'eau avec laquelle on lave un veuf en y introduisant une ou plusieurs feuilles appropriées.

${ }^{\text {a }}$ Depuis une génération environ, de nombreux veufs refusent de porter le pagne noir en attendant un second lavage purificateur au bord de la mer. A Anloga, ils quittent alors le veuvage ce même jour, de la même manière qu'ils le quittaient autrefois à l'issue du second lavage. Se faisant remettre à la plage un cache-sexe et un pagne blancs qu'ils pourront laisser de côté dès le lendemain, ils se parent immédiatement des perles de levée du veuvage et donnent chez eux au retour une petite fête dès le début de laquelle les officiantes les introduisent rituellement au milieu des danseurs. A Atorkor cette suppression de la seconde phase du deuil étant entrée en vigueur avant le raccourcissement de huit à quatre jours de la durée de réclusion à domicile, les veufs continuent de passer quatre jours dans leur chambre, mais durant les quatre jours suivants où ils peuvent occuper la pièce de séjour, ils sont déjà autorisés à ne plus tresser de cordelettes et à recevoir des anis.

${ }^{9}$ A Anloga on autorise dès lors le veuf ou la veuve à se peigner, ce qui reste souvent interdit ailleurs. 
l'épouse défunte qui lui attache sous le genou gauche trois perles godobui (des perles devenues rares, de forme cylindrique, rayées longitudinalement de rouge sur un fond de couleur cendre) dont la réunion est dénommée avhake, ("la guerre s'est ouverte", c'est-àdire est finie). Elles ne lui attachent rien sous le genou droit. Le veuf, s'il le désire, complète lui-même sa tenue en revêtant quelques autres colliers, bracelets, ou bijoux ${ }^{10}$.

Ses parents et ses amis, y ayant été invités, ont déjà commencé à se rassembler dans sa cour et accueillent joyeusement son retour. En arrivant, les deux femmes préposées aux cérémonies prient encore l'esprit de la défunte, déclarant par exemple : "Ton mari t'a fait du bien, il faut que tu lui fasses aussi du bien !". Après avoir commencé à chanter en s'accompagnant des instruments rythmiques gakokoe et axatse, elles font faire quatre tours au veuf au milieu de l'assistance puis, se mettant à danser les premières, l'entraînent à danser à son tour tandis que plusieurs autres personnes se mettent aussi de la partie. Il faut exécuter quatre rythmes différents et chanter, sur chacun de ces rythmes, quatre chansons différentes.

Enfin, le veuf offre de la bière de mil ou de maïs (liha) et de l'alcool aux participants. En retour, chacun lui offre, à titre de cotisation pour l'aider à faire face à ses frais, une petite obole dont le montant n'est pas fixé. Si certains veulent se réjouir encore, ils peuvent continuer à le faire tandis que d'autres se dispersent.

Le veuf pourra porter les perles qui lui ont été remises aussi longtemps qu'il le désirera. Il pourra les enlever dès le lendemain ou les conserver plusieurs semaines ${ }^{11}$.

\footnotetext{
10 Parfois, les perles ne sont remises au veuf qu'à son retour à la maison. Elles diffèrent plus ou moins d'un village à l'autre. Ainsi, à Atorkor, on attache au poignet gauche du veuf une perle rouge sui, une perle bleue gblotsi, un bijou d'or et un grain noir hloku, ce qui semble correspondre aux quatre éléments), à son poignet droit des perles variées, au dessous de son genou gauche une enfilade de petites perles appelées aussi avhake, et au dessous de son genou droit des perles variées.

11 Dans certains villages où le port du pagne noir a été supprimé et où le deuil du veuf prend donc désormais fin avec sa réclusion à domicile, on lui demande parfois, en compensation, de porter ces perles un certain nombre de jours (par exemple, à Atorkor, au minimum 14 jours).
} 


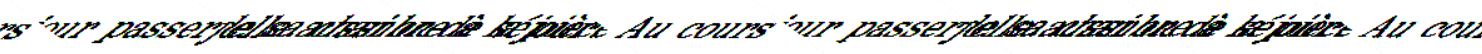

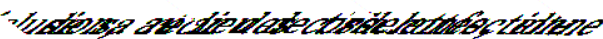

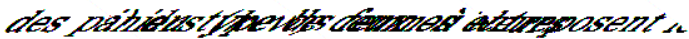

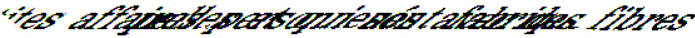

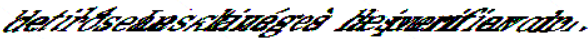

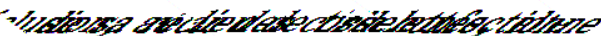

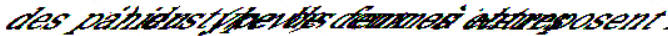

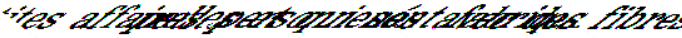

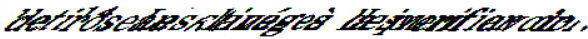

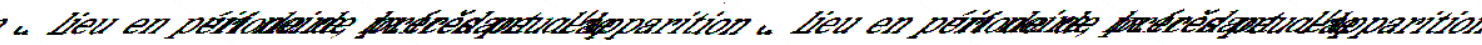

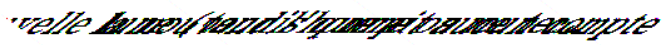

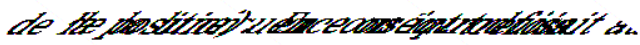

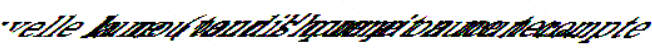

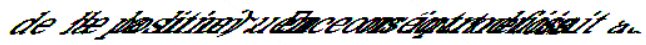

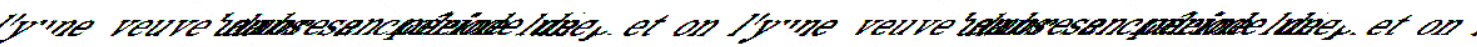

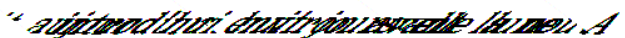

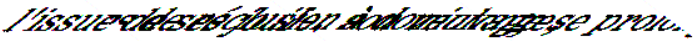

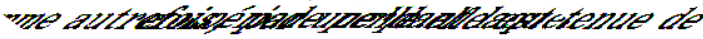

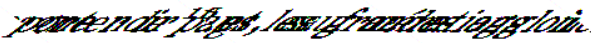

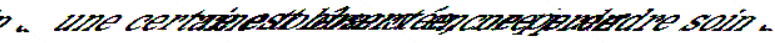

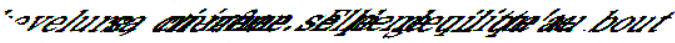

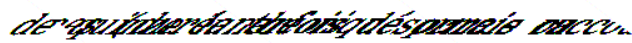

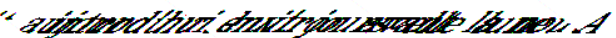

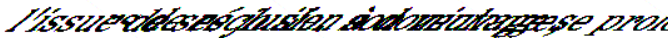

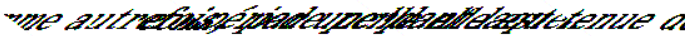

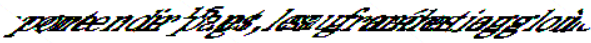

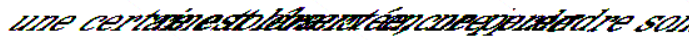

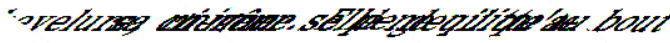

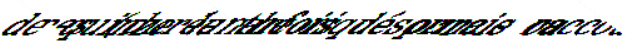

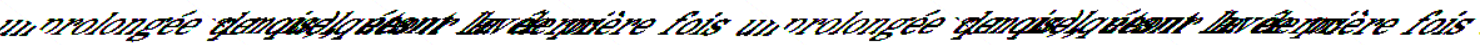

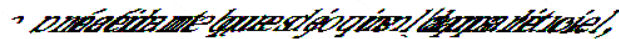

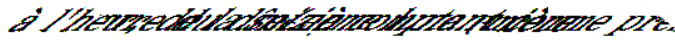

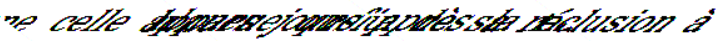

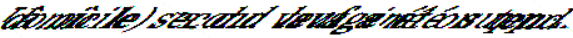

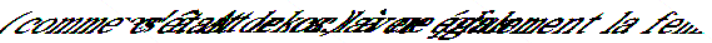

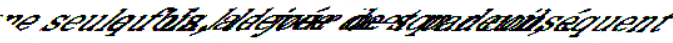

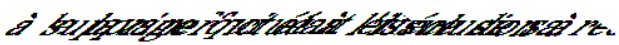

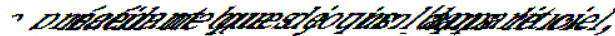

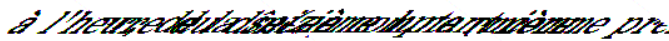

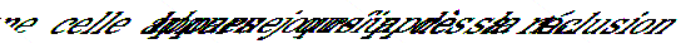

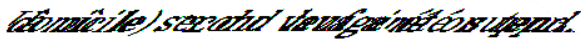

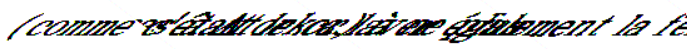

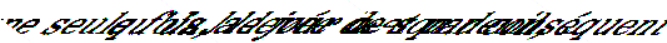

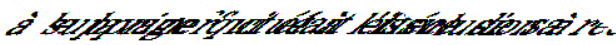

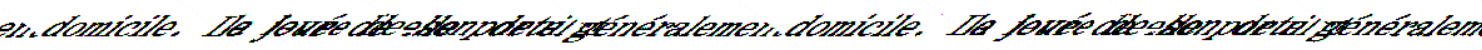

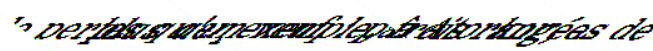

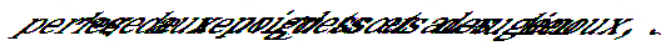

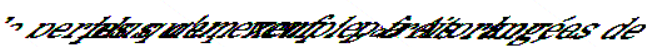

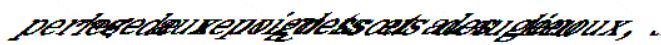

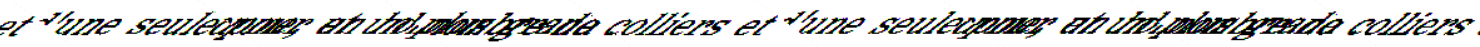

- additzínes.

- adtiazivas.

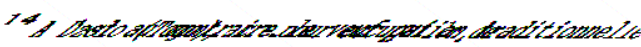

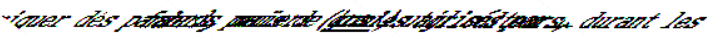

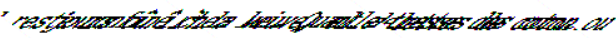

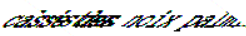

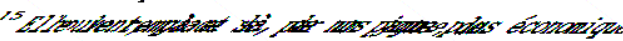

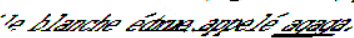

It hato of

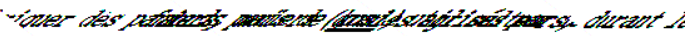

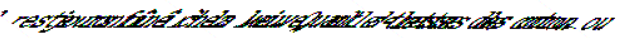
cassis nais arla.

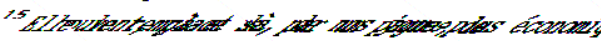

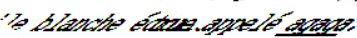




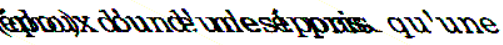

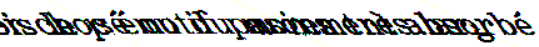

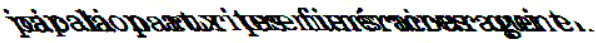

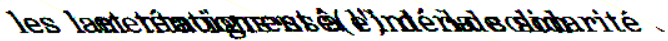
entourage.

mesure

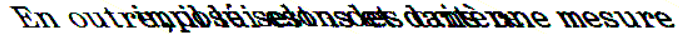

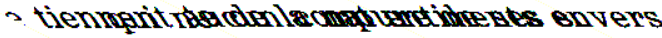

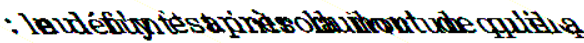

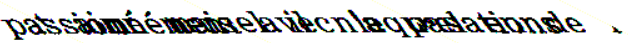

\section{anvers}

Atude quitiala

Magratálande .
Le devil

vernis

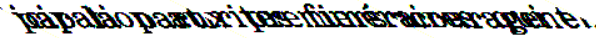

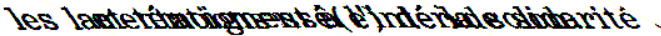
entourage.

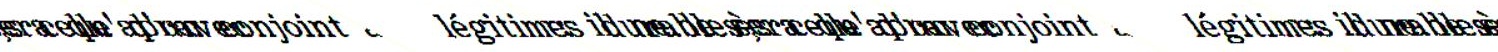

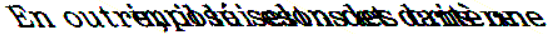

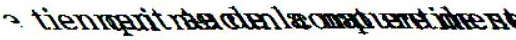

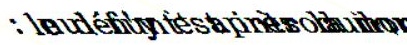

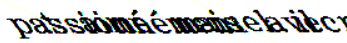

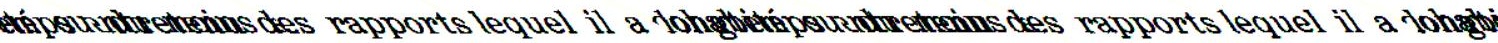
resesese

+ est rédattress gros

+ est redut

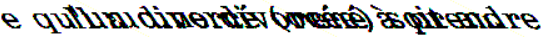

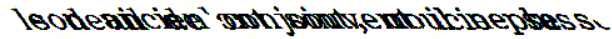

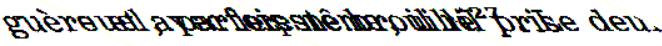

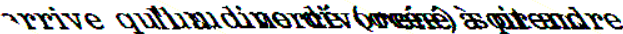

>rriv

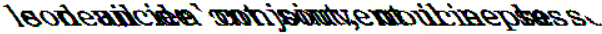

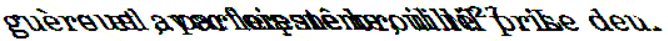

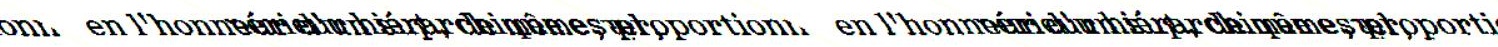

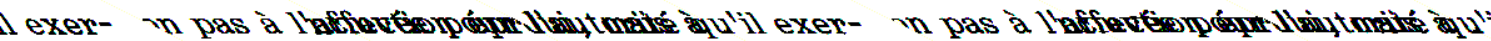

$$
\text { çait. }
$$

átprálotar

reutptobygend dia

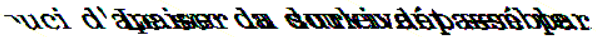

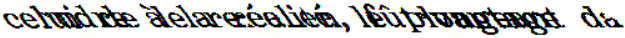

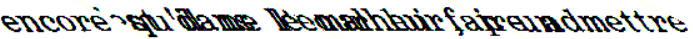
omshthair faireundmettre çait.

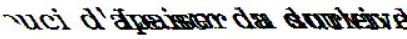

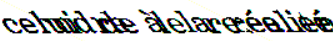
encorè sula da

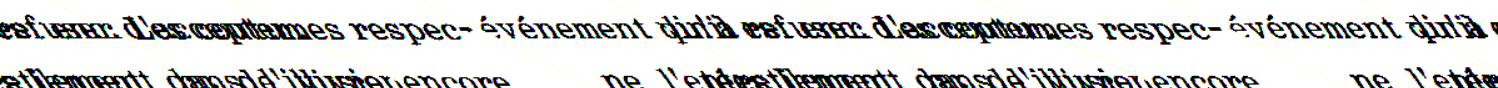

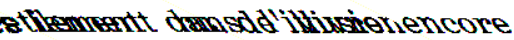

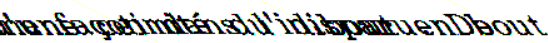

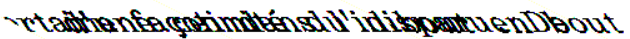

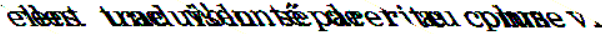

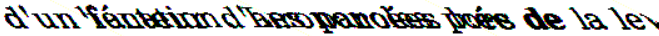

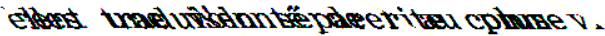

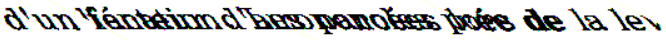

ts

ओंस:

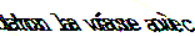

Nistagmenta qualdye IV

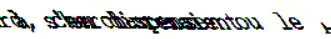
ecomstail Ghara Drolo la

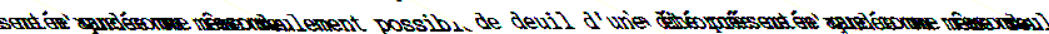
ite par un ar

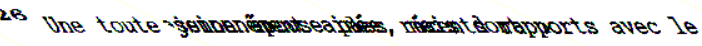

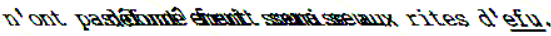

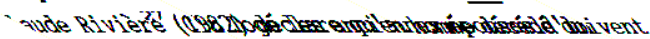

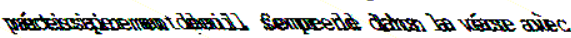

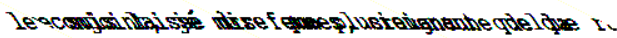

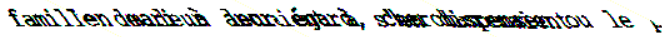

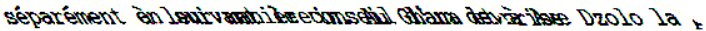
devant etre posimite par un ow

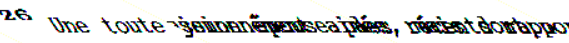

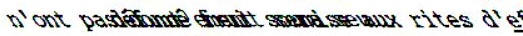

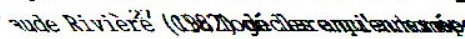

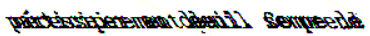

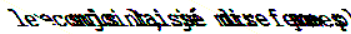

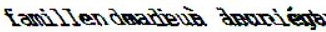
separénent en lewirymiler devant être 
deuil : "A partir d'aujourd'hui vous n'êtes plus mari et femme", pourraient laisser croire que le mariage a été prolongé jusque là en imagination pour donner le temps au veuf ou à la veuve d'en dénouer volontairement les liens plutôt que de subir leur rupture. Cependant les prérogatives du conjoint décédé apparaissent déjà écartées avec insistance avant même la prise de deuil :

Dès après la mort il est prescrit à une veuve de ne jamais abandonner son cache-sexe, de nuit comme de jour, pour éviter toute tentative de jouissance de ses organes sexuels par l'esprit du disparu. Elle ne se permet de l'ôter que très furtivement pour procéder à sa toilette. Claude Rivière (1982) rapporte même que, dans la région d'Anecho, des feuilles odoriférantes d'ahame sont introduites dans le cache-sexe pour en intensifier le pouvoir dissuasif et que des fumigations des mêmes feuilles en complètent l'action, le soir, dans la chambre.

Par ailleurs, pour éviter que l'esprit du disparu ne vienne partager, et donc contaminer dangereusement ou dévitaliser sa nourriture, un veuf ou une veuve prend soin de déposer un morceau de charbon de bois dans sa bouillie du matin, comme dans son plat de viande ou de poisson en sauce, et d'enfoncer une petite baguette pointue de n'importe quel bois dans sa boule de pâte (akplê ou autre). Les piquants blessent les esprits et le charbon de bois (par ailleurs associé à $\mathrm{Gu}$, le dieu du métal et des armes) les souille.

\section{Justification de la prise de deuil}

Le lien avec une personne de l'autre sexe dont la rupture nécessite une prise de deuil (aho) est un lien matrimonial mettant en jeu une sexualité profonde liée aux mystères de la procréation, mélangeant intimement les forces vitales des deux époux, non les "bagatelles" d'ordre sexuel qu'échangent sans lendemain de légers amants.

Les Evhé considèrent en effet qu'un peu de la vie de l'homme subsiste en la femme avec laquelle il a des relations sexuelles sérieuses et peut y être malmené, au point de le rendre malade ou de 
de se vêtir normalement, etc., introduit une rupture grâce à laquelle ses activités, ses amours, ses loisirs, ne seront plus troublés par les rémanences de processus ayant été tranchés nets. Par ailleurs, en le privant de toute occasion de dépenser sa vitalité, elle l'aide fort à propos à en refaire provision. Des rites de lavage achèveront de le convaincre que tout ce qui lui collait fâcheusement à la peau a été renvoyé au pays de l'origine depuis un carrefour, le bord d'une rivière ou celui de l'océan, lui redonnant de l'assurance avant d'affronter à nouveau les difficultés de l'existence.

Pareil traitement rappelle étrangement celui que doit subir un candidat à une autre sorte de nouvelle naissance par initiation à un vodu. Ce ne doit pas être sans raison qu'un veuf ou une veuve est appelé ahosi comme un nouvel initié à un vodu est appelé vhusi ou vodusi. A y regarder de près, la durée idéale du deuil apparaît en effet structurée à l'image de celle d'une initiation.

Tout porte à croire que la réduction de huit à quatre des jours de réclusion du veuf à Anloga fut elle-même précédée d'une réduction de seize à huit jours et que la durée de son confinement était donc autrefois de seize jours (en réalité quinze nuits et quatorze jours). Cette dernière durée est celle dont font état, pour l'homme comme pour la femme, les informations recueillies chez les Anlo de Woe par G.K. Nukunya (1969: 205).

Aussi bien à Atorkor qu'à Anloga, la femme est recluse un nombre de jours qui est toujours un multiple pair du temps de réclusion de l'homme. Nous sommes donc autorisés à estimer que cette durée était théoriquement fixée (comme encore tout récemment à Atorkor) à deux fois seize jours (en réalité trente et une nuits et trente jours). Devant se prolonger encore durant quinze lunaisons entières, la durée totale de son veuvage couvrait dans ces conditions une période approximativement égale à trente-deux demi-lunaisons, soit autant de demi-lunaisons qu'une initiation à Afa compte de tranches de douze heures entre le matin de la réception d'Afa au "bois sacré" par un homme et le matin des premières cérémonies de fin de son initiation (Surgy, 1981, chap. VII, et pp. $306 \& 402$ ), ou 
ornières, il importe aussi de le placer sous la protection d'une divinité appropriée et, par bonheur pour lui, celle-ci ne bornera pas son action à une simple surveillance mais lui offrira, en outre, le moyen de mieux exprimer et cultiver l'aspect de sa nature dont il n'avait pas suffisamment pris soin. L'initiation à Afa va ainsi de pair avec l'adoption d'Afa. De même l'initiation à un vodu, après déconditionnement du sujet des causes de ses troubles par immersion dans ce que Pierre Verger nous décrit comme un "état d'hébétude", est conclue par une consécration solennelle à ce vodu.

Certes le déconditionnement analogue du veuf ou de la veuve ne s'accomplit sous aucun patronage particulier et ne conduit pas à l'adoption d'une divinité du veuvage, mais cela s'explique dans la mesure où le traitement de la personne d'un veuf ou d'une veuve (pour amener le premier à renoncer à la part de sa propre vie emportée par son épouse et la seconde à abandonner la part de vie de son mari dont elle a pris possession) vise essentiellement le conjoint défunt.

Le rite de passage délimité par la prise de deuil (aho) et la levée du deuil permet moins au survivant de retrouver une vie normale qu'il n'assure un bon transfert du défunt d'une condition d'âme insatisfaite agissant inconsidérément pour son propre compte (pouvant par exemple provoquer chez son épouse prématurément remariée une grossesse nerveuse ou une série de grossesses à répétition) à celle d'une âme parfaitement intégrée dans la société de l'autre monde, n'agissant plus qu'en harmonie avec les autres ancêtres ${ }^{32}$. La consécration du sujet à une divinité protectrice paraît remplacée au terme du veuvage par l'installation de l'esprit vital (agbe luvhọ) du défunt auprès de celui de son ancêtre tutélaire (l'amedzọọ) et du principe vital (Kla) ayant présidé à son envoi au monde; en effet il se mettra dès lors à leur disposition pour faire fonction lui aussi

\footnotetext{
32 Claude Rivière (1982) signale que le veuvage est moins long à Notse quand il est pris pour une personne ayant été frappée de "mauvaise mort". Cela peut s'expliquer par le fait que l'âme d'un "mauvais mort" n'a pas à reprendre pied au pays de l'origine en s'y identifiant à une divinité. Tout au plus entre-t-elle, dans le monde intermédiaire où elle continue à évoluer, au service d'une des divinités. Il n'en convient pas moins de l'aider à changer radicalement de rôle.
} 
d'ancêtre tutélaire ou de telle ou telle divinité à laquelle il se trouvera assimilé.

Que conclure des observations précédentes, si ce n'est tout d'abord qu'en raison de leur date indéterminée, souvent tardive, ainsi que de leur indépendance des sentiments ayant été éprouvés pour le défunt, les rites evhé du deuil ne semblent pas pouvoir être considérés comme un traitement social de l'émotion immédiatement ressentie après une mort.

Gardons-nous d'oublier, à ce propos, que les rites du deuil ne font pas partout face au même événement psychologique que celui habituellement occasionné autour de nous par un décès. La fin de la vie d'un proche n'est pas du tout ressentie de la même manière au sein de sociétés dont les institutions et les croyances, notamment celles relatives au statut de la personne humaine, diffèrent considérablement. C'est ainsi qu'en milieu traditionnel evhé (mais je crois que cette observation peut être étendue à une très large partie de l'Afrique noire) on est si persuadé de la subsistance de l'âme d'un mort auprès de ceux qu'il a quittés que sa disparition physique n'est en aucun cas perçue comme un anéantissement ou un éloignement définitif, mais comme une transformation des modalités de sa présence. Bien que toujours difficile à supporter, la mort n'est pas jugée révoltante. Elle fait passer un individu de la condition de personne vivante, appelée à jouer un certain rôle dans le monde, à celle d'un défunt appelé à y jouer un rôle plus éminent encore, car indispensable à l'humanisation de ses descendants ${ }^{33}$. Elle met à la disposition des survivants un agent d'action magique ou un ancêtre supplémentaire à qui ils offriront des cadeaux, de qui ils attendront en retour certaines faveurs, à qui ils rendront des sacrifices, qu'ils associeront à toutes leurs beuveries en leur versant au sol le premier verre de boisson, qu'ils feront parler par l'intermédiaire d'un médium ou he cesseront de consulter chez divers devins pour lui demander conseil et connaître ses exigences. Après qu'elle ait frappé ils se préoccupent

\footnotetext{
33 On peut parfaitement reprendre pour les Evhé ce que j'ai déjà exposé à ce sujet en traitant des Mwaba-Gurma (Surgy 1984).
} 


\section{Raturgmathues}

?. Stuthepr,

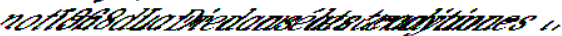

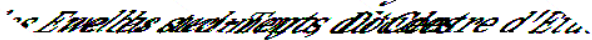

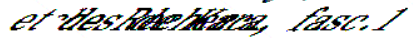

Nituges, Gi.

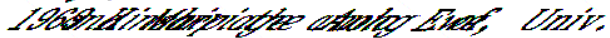

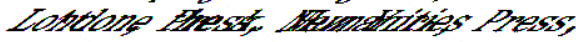
1, 2170

\section{Rivière, Claude}

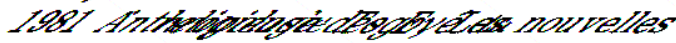

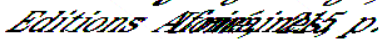

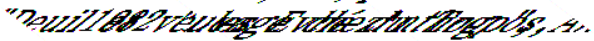
D. HOF,

\section{Alarityses}

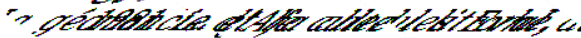

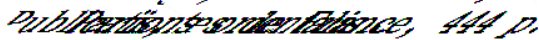

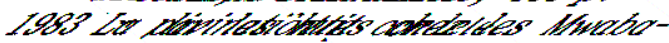

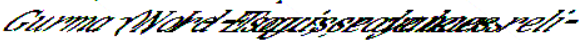

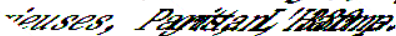

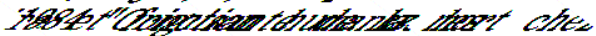

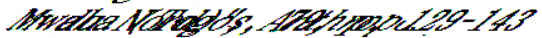

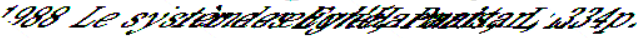

Vegere Pierne

1980 vorists, batrill, 299,
Retrigangatoves

$\because$ shitegre,

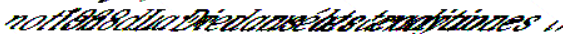

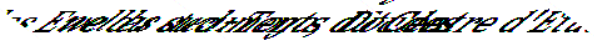

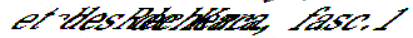

NTSUMYs, Gis.

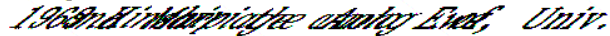

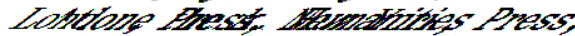
H. $27 \%$ D.

Pivére, Claude

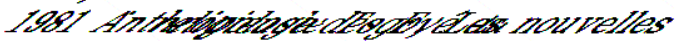

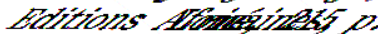

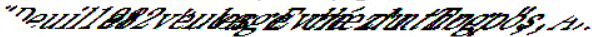

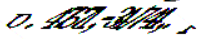

Alaroteges

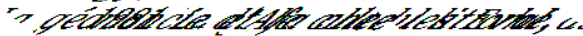

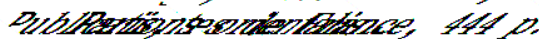

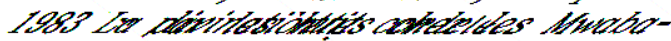

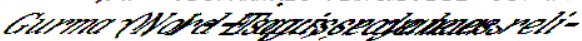

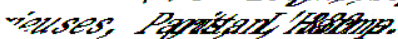

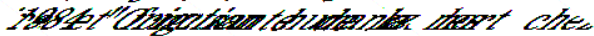

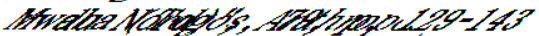

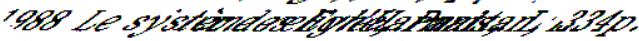

Vergors, Piépre

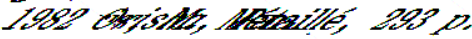


